\title{
The operator-product expansion away from euclidean region
}

\author{
Jan Fischer* and Ivo Vrkoč \\ Institute of Physics, 18221 Prague 8 (J.F.), and Mathematical Institute, 11567 Prague 1 (I.V.), \\ Academy of Sciences of the Czech Republic
}

The role of the operator-product expansion in QCD calculations is discussed. Approximating the two-point correlation function by several terms and assuming an upper bound on the truncation error along the euclidean ray, we consider two model situations to examine how the bound develops with increasing deflection from the euclidean ray towards the cut. We obtain explicit bounds on the truncation error and show that they worsen with the increasing deflection. This result does not support the conventional believe that the remainder is constant for all angles in the complex energy plane. Further refinements of the formalism are discussed.

\section{Introduction}

A standard way to investigate the interconnection between perturbative and non-perturbative effects in quantum chromodynamics is the operator-product expansion (OPE) [1]. In the momentum space, it has the form (we put $y=0$ )

$\mathrm{i} \int \mathrm{d} x \mathrm{e}^{\mathrm{i} q x} A(x) B(0) \approx \sum_{k} C_{k}(q) \mathcal{O}_{k}$

where $q$ is the total four-momentum, $q^{2}=s=$ $-Q^{2}$. The coefficients $C_{k}(q)$ are ordered according to the increasing exponent $k$ in $s^{-k}$.

Unlike perturbative expansions (in powers of the coupling constant $\alpha_{s}$ ), very little is known about the mathematics of the operator product expansion. The knowledge is not sufficient to give (11) precise mathematical meaning, not even is known the exact composition of terms on the right hand side of (11) (in particular, whether terms exponential in the variable $Q^{-1}$ have to be added to describe strong-interaction processes). There are reasons to believe that the series is divergent [2], but it is not known whether it is asymptotic to the function expanded. The largeorder behaviour of its terms is not known either. Last not least, the symbol $\approx$ in (iil) is understood differently in different contexts.

\footnotetext{
*Supported in part by GAAV and GACR (Czech Republic) under grant numbers A1010711 and 202/96/1616 respectively.
}

In spite of serious mathematical uncertainties, the operator product expansion is of paramount importance for solving a number of practical problems in quantum chromodynamics. To calculate many measurable quantities in QCD, it is necessary to investigate the properties of the OPE in the complex $Q^{2}$ plane away from the euclidean region, i.e., away from the semiaxis $Q^{2}>0$. This leads us, with regard to (1), to the problem of finding conditions under which a power expansion of the type

$f\left(1 / Q^{2}\right) \approx \sum_{k} a_{k}(q) / Q^{2 k}$

can be extended to angles away from the euclidean semiaxis in the $Q^{2}$ plane. But such an extension is a delicate problem requiring precise mathematical conditions, which are not known here.

In practical applications, it is usually assumed that the properties of the operator product expansion away from the ray $Q^{2}>0$ are the same as those along it, except for the cut $Q^{2}<0$ (the Minkowski region). Instead of making this simplifying technical assumption, we prefer to introduce some model assumptions which would free the scheme from a priori neglecting the influence of the cut on the truncation error along a ray, keep the model possibly close to real situations and, simultaneously, give the problem precise mathematical meaning. 


\section{The model}

I will discuss the problem on the example of the following model situation. Let $F(s)$ be holomorphic in the complex $s$ plane cut along the positive semiaxis, with the possible exception of a bounded domain around the origin. Let the numbers $a_{k}, k=0,1,2, \ldots n-1$, and a positive number $A_{n}$ exist such that the inequality

$\left|F(s)-\sum_{k=0}^{n-1} a_{k} /(-s)^{k}\right|<A_{n} /|s|^{n}$

holds for all real $s$ smaller than a negative number. The problem is under what conditions this inequality can be continued to the complex plane, and what will be its form away from the original region of validity, i.e. away from the negative real semiaxis (euclidean region).

This problem is of interest for QCD because it could, when solved, tell us how the inequality (3) develops when the ray along which the operator product expansion is studied departs from the euclidean region towards the Minkowski one. We shall discuss the problem under two simplifying assumptions.

Note first that the inequality (3) amounts to assuming that the $n$-th order remainder $R_{n}(-1 / s)$,

$R_{n}(z)=f(z)-\sum_{k=0}^{n-1} a_{k} z^{k}$,

tends to zero for $z \rightarrow 0$ as the $n$-th power of $z$ for at least one value of $n$. (Here, we use the notation $z \equiv \mathrm{e}^{\mathrm{i} \varphi}=-1 / s$ and $f(z) \equiv F(s)$.) This assumption may appear as too optimistic; indeed, it is difficult to make a realiable estimate of the truncation error even in the euclidean region, because it is not known whether there is a limit of QCD in which the operator-product expansion becomes exact.

A second assumption we make is that the logarithmic energy dependence of the expansion coefficients $a_{k}$ can be neglected.

We do not demand, on the other hand, that the series on the left-hand side of (3) be convergent or asymptotic to $F(s)$ : our approach is more general and can be applied whenever the remainder obeys (3) at least for one value of $n$.
Assuming the bound (3), we observe how it varies with the deflection of the ray away from euclidean region. It is natural to expect that the solution to the problem depends on additional assumptions imposed on the function $F(s) \equiv f(z)$. In particular, the form of the dependence of the resulting bound is determined by the assumed character of the discontinuity along the cut.

We consider two possible sets of such additional assumptions.

\section{Results}

(i) In the first of them, we assume that $f(z)$ admits the integral representation of the form

$f(z)=\int_{0}^{\infty} \frac{\rho(t)}{1+z t} \mathrm{~d} t$

with $z$ complex and $\rho(t)$ nonnegative for $t \geq 0$, and that the moment

$a_{n}=\int_{0}^{\infty} t^{n} \rho(t) \mathrm{d} t$

exists for at least one positive integer $n$. Then it follows that the remainders $R_{k}(z)$ as defined by (14), with $k=1,2, \ldots, n$, are bounded by the inequalities

$\left|R_{k}(z)\right| \leq a_{k}|z|^{k}$

and

$\left|R_{k}(z)\right| \leq a_{k}|z|^{k} /|\sin \varphi|$

for $\operatorname{Re} z>0$ and $\operatorname{Re} z<0$ respectively. We see that the denominator on the right-hand side of the inequality (8) produces a looser estimate along rays lying in the halfplane $\operatorname{Re} z<0$, where the cut is present.

Note that the integral representation (5) is not equivalent to the dispersion integral representation that is typical for the QCD two-point functions, because these quantities usually obey a once-subtracted dispersion relation, in which case the moments (6) do not exist. Contrary to this, the approach based on (5) and (6) consists in assuming that at least several lowest moments $a_{n}$ are convergent integrals. This feature may be of interest in some model situations, which we do 
not consider here. To get closer to physics, we consider an alternative scheme below.

(ii) In this second scheme we assume, instead of the above conditions, that $f(z)$ is bounded by a constant $M$ inside a circle of radius $d$ in the cut $z$ plane. The resulting bound on the remainder has the following form:

$\left|R_{n}\left(r \mathrm{e}^{\mathrm{i} \varphi}\right)\right| \leq M_{n}\left(r / d_{n}\right)^{n(1-|\varphi| / \pi)}$.

Here, $M_{n}$ and $d_{n}$ are constants which related to $M$ and $d$. This inequality is a special consequence of a general theorem, which will be published separately [3]. The inequality (9) gives an upper bound on the remainder $R_{n}(z)$ along every ray tending to the origin (i.e., to infinite energy), the estimate becoming worse with increasing deflection from the positive real semiaxis (euclidean region), i.e., with the ray approaching closer the cut.

Since the estimate (9) might seem rather loose (note that $n$ in the exponent on the right hand side is multiplied by an angle-dependent factor that vanishes on the cut), it is perhaps worth mentioning that there are functions that saturate it. Details will be discussed in [3].

\section{Discussion}

The bounds obtained are related to the problem of finding error estimates for the QCD calculations, in which contour integrals of the type

$6 \pi \mathrm{i} \oint_{|s|=m^{2}}\left(1-s / m^{2}\right)^{k}\left(1+2 s / m_{\tau}^{2}\right)^{l} P(s) \frac{\mathrm{d} s}{m^{2}}$

occur (with $k$ and $l$ being nonnegative integers). One example is the determination of $\alpha_{s}(m)$ from the $\tau$ lepton hadronic width [5]; in this case, $m$ is the $\tau$-lepton mass, $k=2, l=1$, and $P(s)$ is a combination of the electromagnetic two-point correlation functions $\Pi(s)$ for the vector and the axial vector colour singlet massless quark currents, the $\Pi(s)$ being defined as

$$
\begin{array}{r}
\Pi^{\mu \nu}=\left(g^{\mu \nu} q^{2}-q^{\mu} q^{\nu}\right) \Pi\left(-q^{2}\right) \\
=\mathrm{i} \int \mathrm{d}^{4} x \mathrm{e}^{-\mathrm{i} q x}\left\langle 0\left|\mathrm{~T}\left(j^{\mu}(x) j^{\nu}(0)\right)\right| 0\right\rangle
\end{array}
$$

for two currents $j^{\mu}$ and $j^{\nu}$. Note that the integral (10) may receive essential contributions from the region near $s=m^{2}$, where the OPE has little chance appropriately to represent the function expanded. A fortunate circumstance is that the double zero of the kinematic factor $\left(1-s / m^{2}\right)^{2}$ in the integrand suppresses the contribution from this dangerous segment. But a quantitative analysis of this argument is, to the best of our knowledge, still lacking.

It has been the motivation of the present research to find an explicit quantitative estimate of the truncation error. In considering the problem of the evaluation of the contour integrals of the type (10), we propose a model scheme of assumptions with the aim to evaluate the influence of the cut $Q^{2}<0$ by estimating the truncation error along different rays in the complex $Q^{2}$ plane. In this scheme, the property (3) occupies the central position. Its combination with analyticity allows an extension of (3) into the complex $s$ plane.

We have cosidered two possible additional assumption sets, (i) (5) and (6), and (ii) the assumption that $f(z)$ is bounded inside a circle of the cut $z$ plane. While the case (i) leads to the bounds (7) and (8), the case (ii) yields the bound (9), in which the exponent itself depends on the phase $\varphi$, thereby representing a significant worsening of the asymptotic bound along rays that are close to the cut.

In case (i), an improvement of the integrated error in the integrals of the type (10) may be reached by reversing the order of the $s$-integration and the $z$-integration. No such chance seems to exist in the case (ii).

Our results can be considered as a step out of the conventional scheme, in which the pragmatic apriori assumption is made that the truncation error is the same along all rays except the cut. Our bounds are based on a plausible, but still crude and fictitious picture of the operator product expansion, possibly good for certain model situations. In application to physics, however, as was pointed out above, the scheme is not fully appropriate either, due to the simplifying assumptions discussed in Section 2 of this talk. A further refinement is desirable. Next step should include a regard to the logarithmic $q$-dependence of the coefficients $a_{k}(q)$. Work along these lines is in progress. 


\section{Acknowledgements}

Helpful discussions on the subject with M. Beneke, I. Caprini, E. de Rafael, S. Narison, M. Neubert and J. Stern are gratefully acknowledged. I am indebted to A. de Rújula for hospitality at the CERN TH Division, and to Stephan Narison for inviting me to this inspiring conference.

\section{REFERENCES}

1. K.G. Wilson, Phys.Rev. 179 (1969) 1499

2. M.A. Shifman, in Continuous Advances in QCD 1994, A. Smilga (ed.), p. 249 (World Scientific, Singapore, 1994) hep-ph/9405246

3. J. Fischer and I. Vrkoč, to be published

4. M. Shifman, Int.J.Mod.Phys. A 11 (1996) 3195

5. E. Braaten, S. Narison and A. Pich, Nucl.Phys. B 373 (1992) 581

A. Pich: QCD tests from tau decays. Invited talk at the 20th Johns Hopkins Workshop (Heidelberg, 27-29 June 1996), hep$\mathrm{ph} / 9701305$

F. LeDiberder and A. Pich, Phys.Lett. B 289 (1992) 165 